\title{
INCIDENCIA DE MORBI-MORTALIDAD MATERNA CON ALTERACIONES HIPERTENSIVA EN EL EMBARAZO Hospital Provincial General Docente Riobamba
}

\author{
Byron Geovanny Hidalgo Cajo ${ }^{1 \text { y } 2}$, Wilson Lizardo Nina Mayancela ${ }^{1}$, \\ Diego Patricio Hidalgo Cajo ${ }^{1}$, Ángel Gualberto Mayacela Alulema ${ }^{1}$, Luis Gerardo Satan Gunza ${ }^{1}$, \\ IVÁN MESÍAS HIDALGO CAJO ${ }^{2}$ \\ ${ }^{1}$ Universidad Nacional de Chimborazo, Ecuador \\ ${ }^{2}$ Escuela Superior Politécnica de Chimborazo, Ecuador
}

PALABRAS CLAVE

Preeclampsia

Trastornos hipertensivos

embarazo

Eclampsia

Síndrome de HELLP
RESUMEN

La investigación identifica la incidencia de la mobi-mortalidad materna con trastornos hipertensivos en el embarazo. Se realizó un estudio Retrospectivo, descriptivo, correlacional y transversal con una muestra de 205 embarazadas. El 94,6\% presentaron preeclamsia y 4,8\% desencadenaron con eclampsia; con relación a la edad el $76 \%$ constan entre 18 y 34 años, 51,7\% presentaron complicaciones antes, durante y después del parto, 45\% con síndrome de HELLP, 9\% retención de restos placentarios y hemorragia postparto y $3 \%$ llegaron a la muerte materna. Se concluye que una buena atención primaria de salud disminuirá la incidencia de alteraciones hipertensivas en el embarazo.
KEY WORDS

Preeclampsia

Pregnancy Hypertensive

Disorders

Eclampsia

HELLP syndrome

\section{ABSTRACT}

Research identifies the incidence of maternal mortality mobi with hypertensive disorders in pregnancy. A retrospective, descriptive, correlational and cross-sectional study with a sample of 205 pregnant was performed. 94.6\% had pre-eclampsia and eclampsia $4.8 \%$ triggered with; with respect to age comprise 76\% between 18 and 34 years, 51.7\% had complications before, during and after childbirth, 45\% with HELLP syndrome, $9 \%$ retention of placental remains and postpartum hemorrhage and reached 3\% maternal death. We conclude that a good primary health care will decrease the incidence of hypertensive disorders in pregnancy. 


\section{Introducción}

$\mathrm{L}$ a preeclampsia es un problema médico de gran importancia debido a su alta morbilidad y mortalidad materna a nivel mundial. Su etiología se desconoce, no obstante se sugiere que posee una base genética e inmunológica que resulta en un trastorno multisistémico caracterizado por hipertensión, bien sea sistólica, diastólica o ambas.

La preeclampsia leve se define como presión arterial mayor o igual a $140 \mathrm{mmHg}$ y diastólica mayor o igual a $90 \mathrm{mmHg}$, mas proteinuria menor a 5 gr en 24h. la preeclampsia severa se define como presión sistólica mayor o igual a $160 \mathrm{mmHg}$ y diastólica igual o mayor a $110 \mathrm{mmHg}$, mas proteinuria mayor o igual a 5 gr en 24 horas, o con daño de órganos diana y alteraciones en los exámenes de laboratorio. Al momento actual, no se dispone de una prueba de detección o una intervención preventiva que sea universal, reproducible y costo-efectiva. El manejo se fundamenta en el diagnóstico y tratamiento oportuno, la prevención de las convulsiones y la interrupción del embarazo. La Preeclampsia es una de las entidades más frecuentes que complica al embarazo, variando su incidencia entre $2 \%$ y $25 \%$ en diferentes partes del mundo, siendo causa contribuyente para numerosas complicaciones graves durante el embarazo, entre ellas el desprendimiento de la placenta normalmente insertada, mortalidad fetal, parto pretérmino; en la madre ocasiona consecuencias muy graves, tales como la insuficiencia renal aguda, hemorragia cerebral, coagulación intravascular diseminada y shock, entre otros. Los riesgos asociados a las convulsiones son la hipoxia severa por las recurrencias, el trauma materno y la neumonía por aspiración. Durante o inmediatamente después del episodio convulsivo deben instalarse medidas de apoyo para evitar lesiones maternas graves y aspiración: proteger la lengua, minimizar el riesgo de aspiración, mantener la vía aérea permeable y administrar oxígeno.

En América Latina la morbilidad materna no se ha reducido sino que ha ido en aumento, ya sea por no tener control del embarazo o por no recibir atención del parto por personal calificado.

Numerosos trabajos científicos hablan de factores epidemiológicos que predisponen a padecer la enfermedad durante el embarazo, tales como las edades extremas de la vida reproductiva de la mujer, bajo nivel escolar, nuliparidad, historia familiar de preeclampsia-eclampsia, obesidad y mujeres delgadas por debajo de su peso normal para la talla.

En el Hospital Provincial General Docente de Riobamba (HPGDR) existen escasa información estadísticos de esta patología:

Como la enfermedad hipertensiva del embarazo aún no se puede evitar, el objetivo de este trabajo es identificar la tasa de incidencia de morbimortalidad materna con alteraciones hipertensivas en el embarazo.

\section{Metodología}

\section{Tipo De Estudio}

Este trabajo es un estudio Retrospectivo, descriptivo, correlacional y transversal porque describe los casos encontrados de Preeclampsia y la morbi-mortalidad materna producida por esta patología.

\section{Área De Estudio}

Comprende el área de Ginecología perteneciente al HPGDR en el periodo Enero - Diciembre 2014

\section{Universo}

El universo del estudio fue seleccionado a través de datos estadísticos de mujeres embarazadas con diagnóstico de preeclampsia existente en el HPGDR.

\section{Muestra}

La muestra fue de 205 casos de mujeres con preeclampsia, considerando los criterios de exclusión.

\section{Criterios de inclusión}

Pacientes que cuenten con diagnóstico de preeclampsia. Pacientes quien acude a la consulta externa de embarazo de alto riesgo.

Pacientes que acepten participar en el estudio.

Pacientes con y sin antecedentes de preeclampsia.

\section{Criterios de exclusión}

Pacientes que no acepten participar en el estudio. Pacientes con trastorno psiquiátrico.

\section{Instrumento de recolección de la información}

Para la recolección de los datos de investigación se elaboró un formulario donde se recogió toda la información necesaria de acuerdo a los objetivos planteados; utilizando como fuente al departamento de Estadística del HPGDR, se elaboró el listado de las historias clínicas de las embarazadas con diagnóstico de Preeclampsia, en el período establecido.

\section{Variables}

\section{Identificación}

\section{Incidencia mortalidad}

- Preeclampsia leve.

- Preeclampsia severa.

- Eclampsia.

- Síndrome de HELLP. 


\section{Operacionalización de la variables}

\begin{tabular}{|l|l|l|}
\hline Variables & Escala & Indicador \\
\hline $\begin{array}{l}\text { Incidencia } \\
\text { mortalidad } \\
\text { leve }\end{array}$ & $\begin{array}{l}\text { Preeclampsiamero de muerte en } \\
\text { mujeres } \\
\text { preeclampsia leve. }\end{array}$ \\
\hline & Preeclampsia & $\begin{array}{l}\text { Número de muerte en } \\
\text { mujeres con } \\
\text { Preeclampsia severa }\end{array}$ \\
\hline & $\begin{array}{l}\text { Síndrome de } \\
\text { HELLP }\end{array}$ & $\begin{array}{l}\text { Número de muerte en } \\
\text { mujeres con eclampsia } \\
\text { mujeres con Síndrome } \\
\text { de HELLP. }\end{array}$ \\
\hline
\end{tabular}

\section{Resultados}

Tabla 1: Edad De La Madre Con Trastorno Hipertensivo Enero - Diciembre 2014

\begin{tabular}{|c|c|c|}
\hline Edad & Número de casos & Porcentaje \\
\hline$<18$ & 23 & 11,2 \\
\hline $18-34$ & 156 & 76,1 \\
\hline $35->35$ & 26 & 12,7 \\
\hline Total & $\mathbf{2 0 5}$ & 100,0 \\
\hline
\end{tabular}

Fuente: Historias Clínicas - Servicio de Ginecología del HPGDR, 2015.

Gráfico 1: Edad De La Madre Con Trastorno Hipertensivo Enero - Diciembre 2014

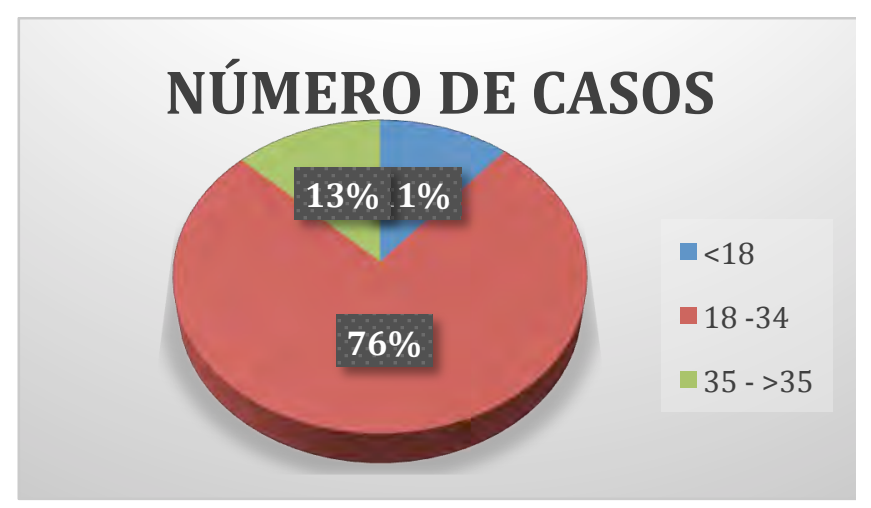

Fuente: Historias Clínicas - servicio de ginecología del HPGDR, 2015.

En el HPGDR se encontraron 205 madres que presentaron algún trastorno hipertensivo del embarazo, de los cuales 23 casos son menores de 18 años, con un porcentaje de $11 \%$; 156 casos de 18 a 34 años con un porcentaje $76 \%, 26$ casos son mayores a 35 años con un porcentaje de $13 \%$. Lo que indica que existe mayor incidencia en mujeres entre 18 a 34 años; que presentan trastornos hipertensivos.
Tabla 2: Tipo De Trastorno Hipertensivo EneroDiciembre 2014

\begin{tabular}{|c|c|c|}
\hline Tipo de trastorno & $\mathrm{N}^{\circ}$ de casos & Porcentaje \\
\hline Preclampsia: & 194 & $94.63 \%$ \\
\hline Eclampsia & 10 & $4.88 \%$ \\
\hline $\begin{array}{l}\text { Hipertensión } \\
\text { gestacional }\end{array}$ & 1 & $0.49 \%$ \\
\hline Total & 205 & $100 \%$ \\
\hline
\end{tabular}

Fuente: Historias Clínicas - servicio de ginecología del HPGDR, 2015

Gráfico 2: Tipo De Trastorno Hipertensivo EneroDiciembre 2014

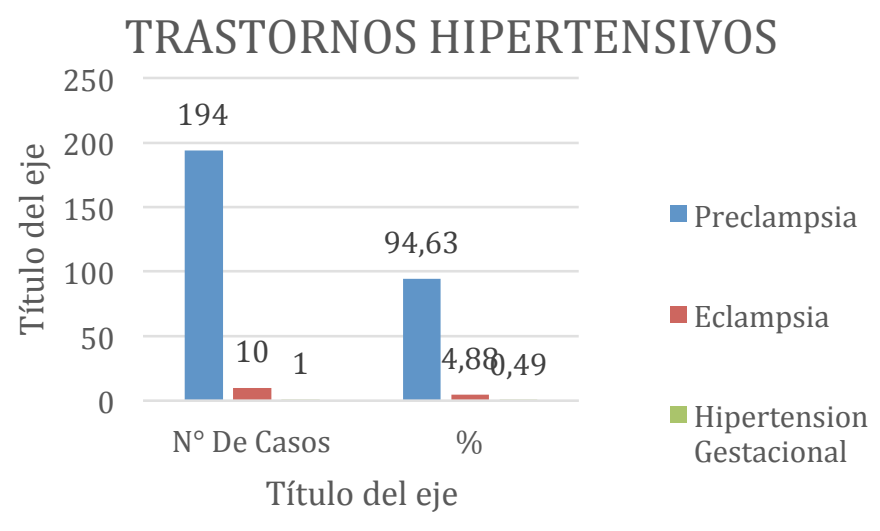

Fuente: Historias Clínicas - servicio de ginecología del HPGDR, 2015.

En el HPGDR se encontraron 205 casos totales de madres con trastornos hipertensivos del embarazo de los cuales 194 pertenecen a preeclampsia, el cual representa un porcentaje de $94.63 \%, 10$ casos de pacientes con Eclampsia que representa un porcentaje de $4.88 \%$ e Hipertensión gestacional 1 caso el cual representa el $0.49 \%$. Lo que indica que de los trastornos hipertensivos del embarazo destaca con una elevada incidencia la preeclampsia.

Tabla 3: Complicaciones en la pre-eclampsia del HPGDR Periodo enero 2014- enero 2016

\begin{tabular}{|c|c|c|}
\hline Complicaciones & Casos & Porcentaje \\
\hline $\begin{array}{c}\text { Sd hellp } \\
\text { placentarios de restos }\end{array}$ & 10 & 45 \\
\hline Oligohidramnios & 2 & 9 \\
\hline $\begin{array}{c}\text { Desprendimiento } \\
\text { normo placentario }\end{array}$ & 6 & 2 \\
\hline RIUC & 1 & 6 \\
\hline Muerte materna & 3 & 3 \\
\hline Hemorragia & 10 & 9 \\
\hline Otros & 26 & 25 \\
\hline & $\mathbf{1 0 6}$ & 100 \\
\hline
\end{tabular}

Fuente: Historias Clínicas - servicio de ginecología del HPGDR, 2015 
Gráfico 3: Complicaciones en la pre-eclampsia del HPGDR Periodo Enero Diciembre 2014

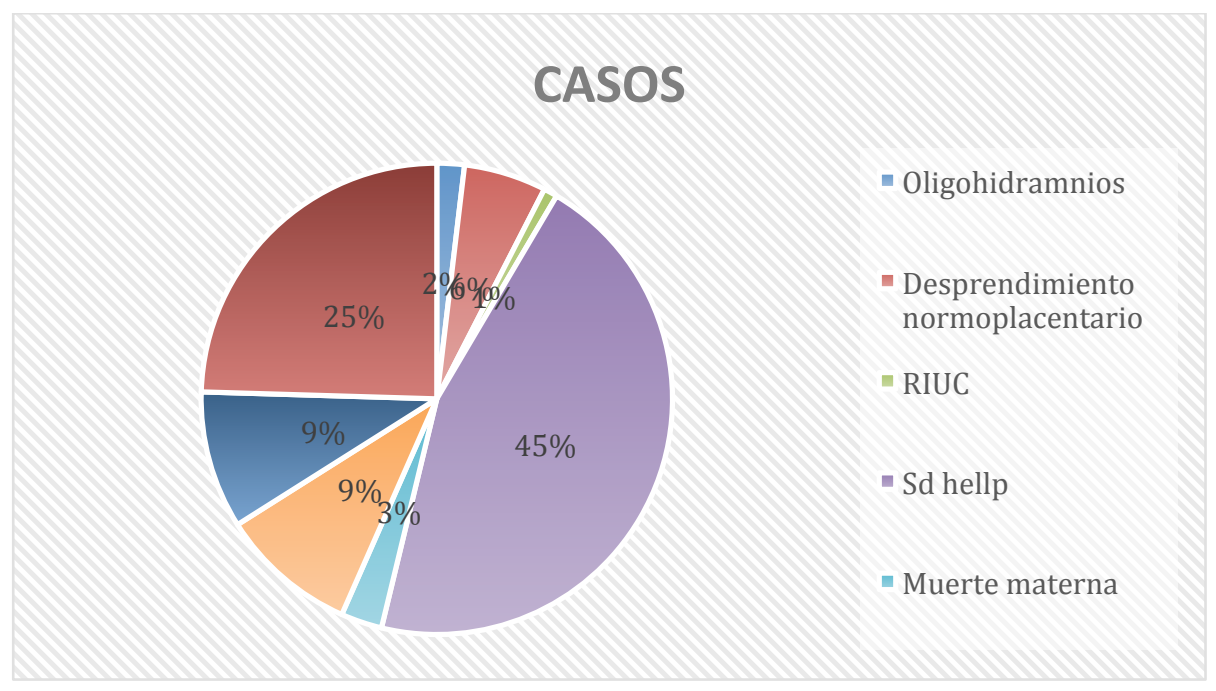

Fuente: Historias Clínicas - servicio de ginecología del HPGDR, 2015.

De los datos analizados por complicaciones presentadas en la pre-eclampsia del HPGDR Periodo enero diciembre 2014, solo en un $3 \%$ han presentado muerte materna y dentro de los casos más frecuentes es decir 48 de un total de 106 (45 $\%)$ es por Síndrome de Hellp, todas las otras complicaciones como son retención de restos placentarios, Oligohidramnios, Desprendimiento normo placentario, RIUC, Hemorragia entre otros se presenta en un porcentaje menor al $10 \%$.
Tabla 4: Vía De Terminación De La Gestación En Pacientes Con Trastornos Hipertensivos Del HPGDR Durante El Periodo Enero Diciembre 2014.

\begin{tabular}{|l|c|c|}
\hline \multicolumn{3}{|c|}{ Vía de terminación de la gestación } \\
\hline Procedimiento & $\mathrm{N}^{\circ}$ casos & $\%$ \\
\hline Cefalovaginal & 56 & $31.1 \%$ \\
\hline Cesárea Total & 124 & $68,8 \%$ \\
\hline \multicolumn{2}{|c|}{180} & $100 \%$ \\
\hline
\end{tabular}

Fuente: Historias Clínicas - servicio de ginecología del HPGDR, 2015

Gráfico 4: Vía De Terminación De La Gestación En Pacientes Con Trastornos Hipertensivos Del HPGDR Durante El Periodo Enero Diciembre 2014.

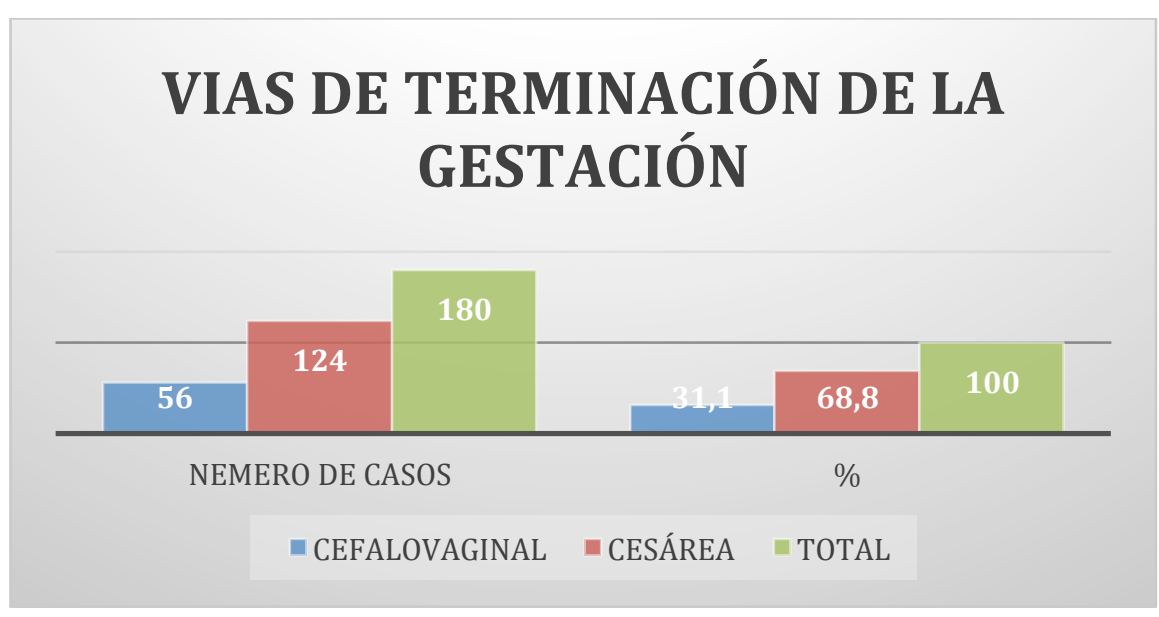

Fuente: Historias Clínicas - Servicio de Ginecología del HPGDR, 2015.

En el HPGDR se encontraron 180 casos de nacimientos en pacientes que presentaron algún Trastorno Hipertensivo en el embarazo, de los cuales 56 de ellos fueron partos normales y 124 nacieron por cesáreas. Esto indica que durante el periodo enero-diciembre 2014 del $100 \%$ de los casos de embarazo con trastornos hipertensivos, el $68.8 \%$ se tuvo que realizar cesárea para evitar complicaciones para el neonato y la madre y solo el $31,1 \%$ se dio por parto normal, siendo lo óptimo y fisiológico para el neonato y la madre. Por lo tanto la mayoría de los trastornos hipertensivos obligan a 
tomar conductas quirúrgicas para salvaguardar la vida e integridad del binomio materno-neonatal.

\section{Discusión}

En el presente estudio investigativo se demuestra la incidencia de Preeclampsia en mujeres menores de 18 años con 23 casos, de un universo de 205 pacientes preeclámpticas de todas las edades. La incidencia de esta patología en este grupo de pacientes jóvenes que se atendieron en el HPGDR es del $11 \%$ de casos en comparación con las de mayor edad con un $13 \%$, lo cual contradice la literatura que manifiesta que la Preeclampsia se desarrolla con más frecuencia en pacientes menores de 18 años. Sin embargo el estudio demuestra que pacientes embarazadas que se encuentran en edades extremas como ser menores de 18 años y mayores de 35 años constituye uno de los principales factores predisponentes para desarrollar Preeclampsia, tal es el caso de las pacientes entre 18 y 34 años con el mayor índice en un $76 \%$ de los casos estudiados.

Según este estudio realizado se determinó que los embarazos preeclámpticas coinciden con otros estudios donde la incidencia en estos grupos de edades se debe; a que las pacientes no acuden a los servicios de salud para recibir orientación adecuada acerca de los diferentes métodos de planificación familiar, antecedentes de preeclampsia, presencia de factores de riesgos y la falta de controles prenatales

Con respecto a las principales complicaciones que presentaron las pacientes preeclámpticas, el estudio reporto que el $51.7 \%$ de total de embarazadas preeclámpticas presentaron diferentes complicaciones; de las cuales el $45 \%$ presento Síndrome de HELLP, 4,8 \% tenían eclampsia, 9\% hemorragia postparto y se apreció en un 3\% de mortalidad materna por esta patología.

Lo que no coincide con los datos estadísticos de un estudio retrospectivo realizado en preeclámpsicas en el HPGDR en el año 2010; en el cual en $77 \%$ del total de embarazada preeclámpsicas no presentaron ninguna complicación antes, durante y después del parto. Además en la literatura reporta que tan solo el 4- 9 $\%$ de las pacientes deben presentar como principal complicación Síndrome de HELLP. Pero en la actualidad se ha disminuido la mortalidad materna por causa de preeclampsia y sus complicaciones; siendo esta complicación de gran preocupación en todos los países del mundo.

\section{Conclusiones}

- $\quad$ En el HPGDR se presentaron 205 casos de pacientes con alteraciones hipertensivas en el embarazo en el periodo de enero diciembre 2014, de los cuales la mayor incidencia corresponde al $76,1 \%$ presente en mujeres entre los 18 a 34 años mientras que el 12,7\% fueron pacientes mayores 35 años.

- Según el tipo de síndrome hipertensivo la preeclampsia presenta 94,6\% correspondiendo al mayor porcentaje, seguido de la eclampsia $4,8 \%$ y $0,4 \%$ la hipertensión gestacional.

- En lo referente a la terminación del embarazo en pacientes que desarrollaron preeclampsia, el 68,8\% corresponde al parto por cesárea y $31,2 \%$ al parto cefalovaginal expuesto en azul y las pacientes con eclampsia el $100 \%$ fue parto por cesárea.

- Las principales complicaciones que se presentaron en la preeclampsia presentes fueron síndrome de HELLP en 45\%, retención de restos placentarios y hemorragia post parto en $9 \%, 6 \%$ por desprendimiento normo placentario y $6 \%$ mientras que en la eclampsia se manifestaron na de las complicaciones fatales fue en $3 \%$ muerte materna. 


\section{Referencias}

[University of Virginia Health System]. «La Hipertensión Inducida por el Embarazo (HIE)» (en español). El Embarazo de Alto Riesgo. Consultado el 20 de diciembre de 2007.

Matthew Warden; B. E. (7 de mayo de 2005). «Preeclampsia (Toxemia of Pregnancy)» (en inglés). eMedicine Obstetrics/gynecology. Consultado el 20 de diciembre de 2007.

Hjartardottir, S., Leifsson, B.G., Geirsson, R.T., Steinthorsdottir, V. (2004). Paternity change and the recurrence risk in familial hypertensive disorder in pregnancy. Hypertens Pregnancy, 23(2), pp. 219-25.

Zhang J. (2007). Partner change, birth interval and risk of pre-eclampsia: a paradoxical triangle. Paediatr Perinat Epidemiol, 21(1), pp. 31-5.

Pacheco Romero, J. (2003). Disfunción endotelial en la preeclampsia. An. Fac. med. [online], 64(1), pp.n43-54.

Burne, J. (2006-01-30). Give Sperm a Fighting Chance. The Times.

Moffett, A., Hiby, S.E. (2007). How does the maternal immune system contribute to the development of preeclampsia? Placenta, 28(A:S), pp. 51-6. 